\title{
Option Pricing Under Stochastic Interest Rates
}

\author{
Haowen Fang
}

School of Business, Sun Yat-sen University, Guangzhou, Guangdong Province, China,510275

\begin{abstract}
This paper reviews the research history of option pricing, then our model assumes that the interest rate subject to a given Vasicek stochastic differential equations, using option pricing by martingale method to study the stochastic interest rate model of European option pricing and obtain the pricing formula. Finally, we compare the differences between the standard European option pricing formulas and European option pricing formula under stochastic interest rate.
\end{abstract}

Index Terms: Option Pricing; Stochastic Interest Rates; Vasicek model, Brownian motions

(C) 2012 Published by MECS Publisher. Selection and/or peer review under responsibility of the Research Association of Modern Education and Computer Science.

\section{Introduction}

Black, Scholes (1973) and Merton[2] (1973) showed in their seminal papers that a derivative security can be priced by creating a replicating portfolio, i.e. a portfolio of primitive securities which matches the payoff of the derivative at maturity. Since both the replication portfolio and the derivative offer the same payoff at maturity, they have to have the same price at any preceding time. Deviations from this equality lead to arbitrage possibilities. Hence, the pricing by duplication procedure inhibits arbitrage by construction. Since then the field of financial engineering has grown phenomenally. The BlackScholes-Merton risk neutrality formulation of the option pricing theory is attractive because the pricing formula of a derivative deduced from their model is a function of several directly observable parameters (except one, which is the volatility parameter). The derivative can be priced as if the market price of the underlying asset's risk is zero.

Detemple[8](2005) reviewed the valuation of American options. Several semi-analytical approximations for American option prices have been proposed in the literature (Barone Adesi and Whaley[1], 1987; Broadie and Detemple[5], 1996; Bunch and Johnson[6], 2000). Although these approaches are fast and accurate, they can not easily be extended beyond the Black-Scholes model. It has been firmly established that the Black-Scholes model is not consistent with quoted option prices. The literature advocates the introduction of stochastic volatility or jump store produce the implied volatility smile observed in the market. The introduction of an additional stochastic volatility factor enormously complicates the pricing of American options. Presently, this can only be done by means of numerical schemes, which involve solving integral equations(Kim[16],1990; Huang, Subrahmanyam, and Yu[13], 1996; Sullivan[20],2000; Detemple and Tian[9],2002), performing Monte Carlo simulations(Broadie and Glasserman[4], 1997; Longstaff and Schwartz[17],2001; Rogers[19], 2002;

* Corresponding author.

E-mail address: fanghaowen@tom.com 
Haugh and Kogan[12],2004), or discrete the partial differential equation(Brennan and Schwartz[3], 1977; Clarke and Parrott[7], 1999; Ikonen and Toivanen[15], 2007). The early exercise premium of the American put option depends on the cost of carry determined by interest rates. Consequently, the volatility of interest rates does affect the decision to exercise this option at any point in time. This fact is recognized in the literature dealing with models with stochastic interest rates(Ho,Stapleton, and Subrahmanyam[11], 1997; Menkveld and Vorst[18],2001;Detemple and Tian[9], 2002). This literature, however, considers only two-factor extensions of the Black-Scholes model assuming that the volatility of the underlying asset is constant.

In this paper, we assume that the interest rate subject to a given Vasicek stochastic differential equations, by using martingale method to study the stochastic interest rate model of European option pricing and obtain the pricing formula.

The paper is organized as follows. In Section 2 we describe the assumptions of the option model, using martingale method, by solving a second order parabolic partial differential equation, we obtain the European option pricing formula. In Section 3 we compare the differences between the standard European option pricing formulas and European option pricing formula under stochastic interest rate.

\section{European option pricing Formula}

The standard BS model makes the following assumptions: the market is frictionless (i. e. no transaction costs or taxes and no penalties for short selling); the market operates continuously, the risk-free interest rate $r$ is a known constant; the asset price $X_{t}$ follows Geometrical Brownian Motion(GBM) with constant volatility $\sigma>0$ and pays no dividends; options and derivatives are European (i. e. no early exercise) and expire at time T with a payoff that depends only on $X_{T}$; the market is arbitrage free.

Under the assumption of GBM, the asset price ${ }^{X_{t}}$ satisfies a stochastic differential equation (sde) of the form

$$
d X_{t}=X_{t}\left(\mu d t+\sigma d B_{t}\right), X_{0}=x_{0}
$$

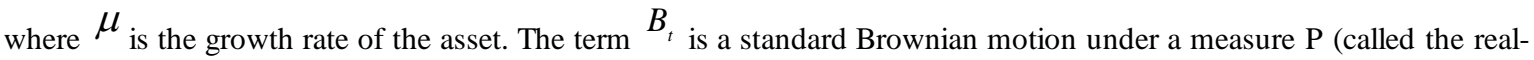
world measure).

Theorem 1 (Itô's Lemma) Let ${ }^{X}$ satisfy the sde $d X_{t}=\alpha(x t) d t+\beta(x, t) d B_{t}$ where $x=X_{t}$ and let $V(x, t)$ be any $C_{2,1}$ function. Then $V\left(X_{t}, t\right)$ satisfies the sde

$$
d V=\left[V_{t}+\alpha V_{x}+\frac{1}{2} \beta^{2} V_{x x}\right] d t+\beta V_{x} d B_{t}
$$

where subscripts on $\mathrm{V}$ denote partial derivatives. It is sometimes more instructive to write this last sde in the equivalent form

$$
d V=\left[V_{t}+\frac{1}{2} \beta^{2} V_{x x}\right] d t+V_{x} d X_{t}
$$

Itô's Lemma is the main tool used to solve sde's. For example, the sde (1) for GBM can be solved by taking $Y_{t}\left(X_{t}\right)=\log \left(X_{t} / x_{0}\right)$. The sde for $Y_{t}$ then becomes $d Y_{t}=\left(\mu-\frac{1}{2} \sigma^{2}\right) d t+\sigma d B_{t}$, and this is readily integrated to give the representation

$$
X_{t} \stackrel{d}{=} x_{0} \exp \left\{\left(\mu-\frac{1}{2} \sigma^{2}\right) t\right\}+\sigma \sqrt{t} Z \quad Z \square N(0,1)
$$

Now, let's deduce the euro-options pricing formula under stochastic interest rate. Assume the asset price $X_{t}$ satisfies 


\section{GBM}

$$
\frac{d X_{t}}{X_{t}}=r_{t} d t+\sigma_{1}\left(r_{t}, t\right) d B_{t}^{1}
$$

The interest rate is given by Vasicek Model[21]

$d r_{t}=a\left(\theta-r_{t}\right) d t+\sigma_{2}\left(r_{t}, t\right) d B_{t}^{2}$

where $\left\{B_{t}^{1}: t \geq 0\right\},\left\{B_{t}^{2}: t \geq 0\right\}$ are standard Brown motions,

$\operatorname{cov}\left(d B_{t}^{1}, d B_{t}^{2}\right)=\rho d t \quad(|\rho|<1)$

Let $V_{t}=V\left(X_{t}, r_{t}, t\right)$ denote the price of the call European option,

$V_{t}=\left(X_{t}-K\right)^{+}, K_{\text {is }}$ the strike price.

We will find the option price. Using $\Delta-$ hedging technical we derive function $V\left(X_{t}, r_{t}, t\right)$ satisfy the appropriate sde, and obtain the portfolio

$$
\Pi_{t}=V_{t}-\Delta_{1 t} X_{t}-\Delta_{2 t} P_{t}
$$

Choose $\Delta_{1 t}$ share of stock and $\Delta_{2 t}$ share of zero-coupon, the portfolio $\prod_{t}$ is risk-free in the period ${ }^{[t, t+d t]}$.It's also mean if choose appropriate $\Delta_{1 t}$ and $\Delta_{2 t}$, then we can get

$d \prod_{t}=d V_{t}-\Delta_{1 t} d X_{t}-\Delta_{2 t} d P_{t}$,

which is risk-free, and then

$d \prod_{t}=r_{t} \Pi_{t} d t=r_{t}\left[V_{t}-\Delta_{1 t} X_{t}-\Delta_{2 t} P_{t}\right] d t$

Here $P_{t}=P\left(r_{t}, t ; T\right)$ is the price of zero-coupon, and satisfies a stochastic differential equation (sde) of the form[22]

$\frac{d P_{t}}{P_{t}}=r_{t} d t-X_{V}(t) \sigma d B_{t}$

(Vasicek Model)

or

$$
\frac{d P_{t}}{P_{t}}=r_{t} d t-X_{C}(t) \sigma \sqrt{r_{t}} d B_{t}
$$

By Itô's Lemma, (4) can be written as

\section{(C-I-R Model).}

$$
\begin{aligned}
d \Pi_{t}= & {\left[\frac{\partial V}{\partial t}+\frac{1}{2} \sigma_{1}^{2} X^{2} \frac{\partial^{2} V}{\partial X^{2}}+\sigma_{1} \sigma_{2} \rho X \frac{\partial^{2} V}{\partial X \partial r}+\frac{1}{2} \sigma_{2}^{2} \frac{\partial^{2} V}{\partial r^{2}}\right] d t } \\
& +\left(\frac{\partial V}{\partial X}-\Delta_{1 t}\right) \mathrm{d} X_{t}+\left(\frac{\partial V}{\partial r}-\Delta_{2 t} \frac{\partial P}{\partial r}\right) d r_{t} \\
& -\Delta_{2 t}\left(\frac{\partial P}{\partial t}+\frac{1}{2} \sigma_{2}^{2} \frac{\partial^{2} P}{\partial r^{2}}\right) d t
\end{aligned}
$$

The third term of the right equation can be substitute as

$$
-\Delta_{2 t}\left(r P-a\left(\theta-r \frac{\partial P}{\partial r}\right)\right) d t
$$

In order to eliminate the risk, let

considering (7) and (2), we obtain

$$
\frac{\partial V}{\partial X}=\Delta_{1 t}, \quad \Delta_{2 t}=\frac{\partial V}{\partial t} / \frac{\partial P}{\partial r},
$$




$$
\begin{gathered}
\frac{\partial V}{\partial t}+\frac{1}{2} \sigma_{1}^{2} X^{2} \frac{\partial^{2} V}{\partial X^{2}}+\sigma_{1} \sigma_{2} \rho X \frac{\partial^{2} V}{\partial X \partial r}+\frac{1}{2} \sigma_{2}^{2} \frac{\partial^{2} V}{\partial r^{2}}+r X \frac{\partial V}{\partial X} \\
+a(\theta-r) \frac{\partial V}{\partial r}-r V=0 \quad\left(r \in \square, X \in \square^{+}, t \in[0, T]\right) \\
\text { as } t=T, V(X, t, T)=(X-K)^{+} \quad\left(r \in \square, X \in \square^{+}\right)
\end{gathered}
$$

We know by the martingale method option pricing theory[10], there exist a martingale measure $Q$, such that

$$
V_{t}=E^{Q}\left(e^{-\int_{t}^{T} r_{\tau} d \tau}\left(X_{T}-K\right)^{+} \mid r(t)=r_{t}, X(t)=X_{t}\right)
$$

To transform valuation of unit of account, that zero-coupon $P_{t}=P\left(r_{t}, t ; T\right)$ as a new unit of account, and a corresponding price system introduced

$$
X_{t}=\frac{X_{t}}{P_{t}}, \quad V_{t}=\frac{V_{t}}{P_{t}}
$$

Based on unit conversion valuation theory, the equivalent martingale measure exists, such that (10) can be rewritten as

$$
\begin{aligned}
V_{t} & =E^{Q^{U}}\left(V_{t} \mid r(t)=r_{t}, X(t)=X_{t}\right) \\
& =E^{Q^{U}}\left(\left(\frac{X_{T}}{P_{T}}-K\right)^{+} \mid P(t)=P_{t}, X(t)=X_{t}\right) \\
& =E^{Q^{U}}\left(\left(X_{T}-K\right)^{+} \mid P(t)=P_{t}, X(t)=X_{t}\right)
\end{aligned}
$$

In the last equation, we use the fact $P_{T}=1$.

(12) showed that the proceeds at the time $T$, function $V_{t}$ can be transformed into only depends on $X_{t}$. In the Vasicek model, zero-coupon price processes satisfies stochastic differential equation,

$$
\frac{d P_{t}}{P_{t}}=r_{t} d t-M(t) \sigma d B_{t}
$$

According to (1),

so,

$$
d \ln P_{t}=\left[r_{t}-\frac{1}{2} \sigma_{2}^{2} X_{V}^{2}(t)\right] d t-\sigma_{2} X_{V}(t) d B_{t}^{2}
$$

$$
d \ln X_{t}=\left[r_{t}-\frac{1}{2} \sigma_{1}^{2}\right] d t+\sigma_{1} d B_{t}^{2}
$$

As for $X_{t}=\frac{X_{t}}{P_{t}}$,such that

$$
\begin{aligned}
d \ln X_{t} & =d \ln X_{t}-d \ln P_{t} \\
& =\frac{1}{2}\left[\sigma_{2}^{2} X_{V}^{2}(t)-\sigma_{1}^{2}\right] d t+\sigma_{1} d B_{t}^{1}+\sigma_{2} X(t) d B_{t}^{2}
\end{aligned}
$$

This shows that for the Vasicek model, the stochastic differential equations of $X_{t}$ is no longer significant contain with $X_{t}$ and $r_{t}$, so we can put (12) rewritten as 


$$
V_{t}=E^{Q^{U}}\left(\left(X_{T}-K\right)^{+} \mid X(t)=X_{t}\right)
$$

This shows that for the new price system $\left\{X_{t}, V_{t}\right\}$, function correlation $V_{t}=V\left(X_{t}, t\right)$ holds.

To solve problem (8) and (9), we draw a new transformation of independent variables,

$$
y=\frac{X}{P(r, t ; T)}
$$

and a new unknown function denotes as

$$
V(y, t)=\frac{V(X, r, t)}{P(r, t ; T)}
$$

According to primarily computations,

$$
\begin{aligned}
& \frac{\partial V}{\partial t}=V \frac{\partial P}{\partial t}+P \frac{\partial V}{\partial t}-y \frac{\partial V}{\partial y} \frac{\partial P}{\partial t} \\
& \frac{\partial V}{\partial r}=V \frac{\partial P}{\partial r}-y \frac{\partial V}{\partial y} \frac{\partial P}{\partial r} \\
& \frac{\partial V}{\partial X}=\frac{\partial V}{\partial y} \\
& \frac{\partial^{2} V}{\partial r^{2}}=V \frac{\partial^{2} P}{\partial r^{2}}-y \frac{\partial V}{\partial y} \frac{\partial^{2} P}{\partial r^{2}}-y^{2} \frac{\partial^{2} V}{\partial y^{2}} \frac{1}{P}\left(\frac{\partial P}{\partial r}\right)^{2} \\
& \frac{\partial^{2} V}{\partial r \partial X}=-y \frac{\partial^{2} V}{\partial y^{2}} \frac{1}{P} \frac{\partial P}{\partial r} \\
& \frac{\partial^{2} V}{\partial X^{2}}=\frac{1}{P} \frac{\partial^{2} V}{\partial y^{2}}
\end{aligned}
$$

Substitute them into (8), and divided by $P(r, t ; T)$, such that

$$
\begin{aligned}
\frac{\partial V}{\partial t}+ & \frac{1}{2}\left[\sigma_{1}^{2} \frac{X^{2}}{P^{2}}-2 \sigma_{1} \sigma_{2} \rho \frac{X}{P} \frac{\partial P}{\partial r}+\frac{1}{2} \sigma_{2}^{2} y^{2}\left(\frac{1}{P} \frac{\partial P}{\partial r}\right)^{2}\right] \frac{\partial^{2} V}{\partial y^{2}} \\
& +\frac{1}{P}\left[\frac{\partial P}{\partial t}+\frac{\sigma_{2}^{2}}{2} \frac{\partial^{2} P}{\partial r^{2}}+a(\theta-r) \frac{\partial P}{\partial r}-r \frac{X}{y}\right] y \frac{\partial V}{\partial y} \\
& +\frac{1}{P}\left[\frac{\partial P}{\partial t}+\frac{\sigma_{2}^{2}}{2} \frac{\partial^{2} P}{\partial r^{2}}+a(\theta-r) \frac{\partial P}{\partial r}-r P\right] V=0
\end{aligned}
$$

Considering transformation (13) and the function $P(r, t ; T)$ satisfies the following second order parabolic pde's Cauchy problem

$$
\left\{\begin{array}{l}
\frac{\partial P}{\partial t}+\frac{\sigma^{2}}{2} \frac{\partial^{2} P}{\partial r^{2}}+a(\theta-r) \frac{\partial P}{\partial r}-r P=0 \quad(r \in \square, t \in[0, T]) \\
P(r, T)=1
\end{array}\right.
$$

Then, we immediately find that function $V(y, t)$ satisfies the equation

\footnotetext{
Then, we inmedrately find that function $V(y, t)$ satisfies the equation
} 
and the definitely solution condition is

$$
\begin{gathered}
\frac{\partial V}{\partial r}+\frac{1}{2} \sigma^{2}(t) y^{2} \frac{\partial^{2} V}{\partial y^{2}}=0 \\
V(y, T)=\frac{V(X, r, T)}{P(r, T ; T)}=(y-K)^{+}
\end{gathered}
$$

where $K$ is the option's strike price,

$$
\begin{aligned}
& \sigma(t)=\sqrt{\sigma_{1}^{2}+2 \rho \sigma_{1} \sigma_{2} X(t)+\sigma_{2}^{2} X^{2}(t)} \\
& X(t)=-\frac{1}{P_{t}} \frac{\partial P}{\partial r}
\end{aligned}
$$

The solution of problem (15) can be expressed by the general Black-Scholes formula

$$
\begin{aligned}
& V(y, t)=y N\left(d_{1}\right)-K N\left(d_{2}\right) \\
& d_{1}=\frac{\ln \frac{y}{K}+\frac{1}{2} \int_{t}^{T} \sigma^{2}(\tau) d \tau}{\sqrt{\int_{t}^{T} \sigma^{2}(\tau) d \tau}} \\
& d_{2}=d_{1}-\sqrt{\int_{t}^{T} \sigma^{2}(\tau) d \tau}
\end{aligned}
$$

Reverse to the original variables $X, r, t$ and unknown function $V$ by the transformation (13) and (14), such that (16) and (17) become

$$
\begin{array}{r}
V(X, r, t)=P(r, t ; T) V\left(\frac{X}{P(r, t ; T)}, t\right) \\
=X N\left(d_{1}^{*}\right)-K P(r, t ; T) N\left(d_{2}^{*}\right) \\
d_{1}^{*}=\frac{\ln \frac{X}{K}-\ln P(r, t ; T)+\frac{1}{2} \int_{t}^{T} \sigma^{2}(\tau) d \tau}{\sqrt{\int_{t}^{T} \sigma^{2}(\tau) d \tau}} \\
d_{2}^{*}=d_{1}^{*}-\sqrt{\int_{t}^{T} \sigma^{2}(\tau) d \tau}
\end{array}
$$

This formula was first proposed by Merton in 1973, when he was not received the random model of shortterm interest rate $r$, but directly starting from the zero-coupon $P_{t}$, assuming $P_{t}$ to meet the geometric Brownian motion, under the martingale measure, it is described by the following Stochastic differential equations,

$$
\frac{d P_{t}}{P}=r d t+\sigma_{P} d B_{t}^{1}
$$

where $\left\{B_{t}^{1}: t \geq 0\right\}$ is standard Brown motion, $\sigma_{P}$ is the zero-coupon's (bonds) volatility.

Thus, European call option pricing formula is

$V(X, P, t)=\mathrm{XN}\left(d_{1}^{*}\right)-K P N\left(d_{2}^{*}\right)$

which 


$$
\begin{aligned}
& d_{1}^{*}=\frac{\ln \frac{X}{K}-\ln P+\frac{1}{2} \int_{t}^{T} \sigma^{2}(\tau) d \tau}{\sqrt{\int_{t}^{T} \sigma^{2}(\tau) d \tau}} \\
& d_{2}^{*}=d_{1}^{*}-\sqrt{\int_{t}^{T} \sigma^{2}(\tau) d \tau} \\
& \sigma^{2}(\tau)=\sigma_{1}^{2}+\sigma_{P}^{2}-2 \rho \sigma_{1} \sigma_{P}
\end{aligned}
$$

\section{Conclusions}

Compared with the standard European option pricing formula and European option under stochastic interest rate, there are only two differences: one is zero-coupon replaced by $e^{-r(T-t)}$; another is that using $\sigma$ instead of stock price volatility $\sigma_{1}$. Except that the pricing formulas is exactly the same form.

Analysis from the actual markets, $\sigma_{P} \square \sigma_{1}$ means zero-coupon's (bonds) volatility is far smaller than stock market's volatility, but in general $\sigma_{P}=\sigma_{P}(t), \sigma_{P}(t)$ is monotonic decreasing, and $\lim _{t \rightarrow T} \sigma_{P}(t)=0$. $\sigma_{\text {and }} \sigma_{1}$ in fact has the minor difference. Because in general, stock prices and bond prices are positively correlated, then $\rho>0$. Therefore, if $\sigma_{P}<2 \rho \sigma_{1}$, by equation (24) we know $\sigma<\sigma_{1}$ holds. Therefore under stochastic interest rates, the price of an option but have slightly decreased.

If a short-term interest rate model is given, only for the Vasicek model and Hull-White model[14], European option pricing formula has a simple form of the Merton formula (23). For C-I-R model, the corresponding zero-coupon stochastic model, the fluctuations in the rate of entry also including $\sqrt{r_{t}}$, so it can not write (22), so by pricing unit conversion of lower dimension than Number of purposes, such as lost that possible style of (24).

\section{References}

[1]Barone-Adesi, G., Whaley, R., Efficient analytical approximation of American option values. Journal of Finance 42, 1987,301-320.

[2]Black, F., Scholes, M., The pricing of options and corporate liabilities. Journal of Political Economy 81, 1973, 637-654.

[3]Brennan, M., Schwartz, E., The valuation of American put options. Journal of Finance 32, $1977,449-462$.

[4]Broadie, M., Glasserman, P., Pricing American-style securities using simulation. Journal of Economic Dynamics and Control 21, 1997,1323-1352.

[5]Broadie, M., Detemple, J. American option valuation: new bounds, approximations, and a comparison of existing methods. Review of Financial Studies 9, , 1996,211-1250.

[6]Bunch, D., Johnson, H., 2000. The American put option and its critical stock price. Journal of Finance 55, 2333-2356.

[7]Clarke, N., Parrott, K., Multigrid for American option pricing with stochastic volatility. Applied Mathematical Finance 6, 1999,177-195.

[8]Detemple, J.,. American-Style Derivatives: Valuation and Computation. Chapman \& Hall/CRC Financial Mathematics Series, Boca Raton, FL. 2005

[9]Detemple, J., Tian, W., The valuation of American options for a class of diffusion processes. Management Science 48, 2002,917-937. 
[10]Geman H., N El Karoui , Changes of Numeraire, changes of probability measure and option pricing. Journal of Applied Probability, 32, 1995,443-458

[11]Ho, T., Stapleton, R., Subrahmanyam, M., The valuation of American options with stochastic interest rates: a generalization of the Geske-Johnson technique. Journal of Finance 52, 1997. 827-840.

[12]Haugh, M., Kogan, L., 2004. Pricing American options: a duality approach. Operations Research 52, 258270.

[13]Huang, J., Subrahmanyam, M., Yu, G., Pricing and hedging American options: a recursive integration method. Review of Financial Studies 9, 1996,27-300.

[14]Hull, J., White, A., Pricing interest rate derivatives securities, Review of Financial Studies,3, 1990,573-592

[15]Ikonen, S., Toivanen, J., Efficient numerical methods for pricing American options under stochastic volatility. Numerical Methods for Partial Differential Equations 24, 2007, 104-126.

[16]Kim, I., The analytic valuation of American options. Review of Financial Studies 3, 1990,547-572.

[17]Longstaff, F., Schwartz, E., Valuing American options by simula- tion: a simple least-squares approach. Review of Financial Studies 14, 2001, 113-147.

[18]Menkveld, A., Vorst, T., A pricing model for American options with Gaussian interest rates. Annals of Operations Research 100, 2001,211-226.

[19]Rogers, L., Monte Carlo valuation of American options. Mathematical Finance 12, 2002,271-286.

[20]Sullivan, M., Valuing American put options using Gaussian quadrature. Review of Financial Studies 13, 2000,75-94.

[21]Vasicek Oldrich, An equilibrium characterization of term structure, Journal of Financial Economics,5, 1977,177-188. 